may in part correspond to previously reported sequences. The absence of some fragments in healthy control joints is indirect evidence for differentially upregulated TNF-a peptide variants in arthritis.

\section{THU0029 EFFECT OF IL-4 ON IL-1 INDUCED INTRACELLULAR CA2+ CHANGS AND INHIBITION OF PG SYNTHESIS IN ARTICULAR CHONDROCYTES}

${ }^{1} \mathrm{~F}$ Nishisaka, ${ }^{2} \mathrm{~K}$ Fukuda, ${ }^{3} \mathrm{~S}$ Soenn, ${ }^{1} \mathrm{M}$ Saito, ${ }^{1} \mathrm{M}$ Oh, ${ }^{1} \mathrm{~T}$ Matsushita, ${ }^{1} \mathrm{H}$ Kikuchi. 'Orthopaedic Surgery, Sakai Hospital, Kinki University, School of Medicine, Sakai; ${ }^{2}$ Orthopaedic Surgery, Kinki University, School of Medicine, Osakasayama; ${ }^{3}$ Orthopaedic Surgery, Nara Hospital, Kinki University, School of Medicine, Ikoma, Japan

\subsection{6/annrheumdis-2001.826}

Background Studies suggest that interleukin-1 (IL-1) is the key mediator of arthritis and we have investigated the effect of IL-1 on cartilage degradation. We demonstrated that IL-1 inhibited proteoglycan (PG) synthesis via enhanced nitric oxide (NO) synthesis by chondrocytes. We also demonstrated that IL-1 increased intracellular $\mathrm{Ca}^{2+}$ ion concentration $\left(\left[\mathrm{Ca}^{2+}\right] \mathrm{i}\right)$ of chondrocytes. On the other hand, interleukin-4 (IL-4) is a T-cell derived 20 $\mathrm{kDa}$ glycoprotein and antagonise the cytokine mediated cartilage degradation.

Objectives We examined the effect of IL-4 on IL-1-enhanced $\mathrm{NO}$ synthesis in parallel with intracellular Ca levels and PG synthesis.

Methods Bovine articular chondrocytes were obtained. PG synthesis was measured with [35S] sulfate incorporation. NO levels were measured by use of an $\mathrm{NO}$ chemiluminescence analyzer. $\mathrm{Ca}^{2+}$ imaging was carried out with Fura2-AM loaded chondrocyte using the digital fluorescence-imaging system.

Results In the presence of IL-4, IL-1-enhanced NO release was completely abolished. IL-4 functions mainly as anti-inflammatory, as well as IL-10 and IL-13. To our knowledge, this is the first report demonstrating an inhibitory effect of IL-4 in NO release stimulated by IL-1. Alterations in $\left[\mathrm{Ca}^{2+}\right] \mathrm{i}$ provide a ubiquitous cell signalling system that mediates a variety of cellular process. IL-1 evoked an increase in the levels of $\left[\mathrm{Ca}^{2+}\right] \mathrm{i}$, a response characterised by a steep onset and followed by a slower decline to preexisting values. IL-4 also abolished the IL-1induced $\left[\mathrm{Ca}^{2+}\right] \mathrm{i}$ increase in the chondrocytes. We examined the effect of IL-4 on PG synthesis. Although IL-4 did not affect PG synthesis, IL-1-inhibited PG synthesis was reversed with the addition of IL-4. It is possible that IL-4 reversed the IL-1-inhibited PG synthesis through the inhibition of NO synthesis and $\left[\mathrm{Ca}^{2+}\right] \mathrm{i}$ increase.

Conclusion IL-4 plays a chondroprotecting effect antagonising with IL-1-inhibited PG synthesis.

\section{REFERENCES}

1 Yeh LA, Augustine AJ, Lee P, Riviere LR, Sheldon A. Interleukin-4, an inhibitor of cartilage breakdown in bovine articular cartilage explants. J Rheumatol. 1995;22 (9):1740-6

2 Fukuda K, Kumano F, Takayama M, Saito M, Otani K, Tanaka S. Zonal differences in nitric oxide synthesis by bovine chondrocytes exposed to interleukin-1. Inflamm Res. 1995;44(10):434-7
THU0030 INCREASED COLLAGEN PRODUCTION BY HUMAN LUNG FIBROBLASTS IN THE RESPONSE TO TUMOUR NECROSIS FACTOR-RELATED APOPTOSIS-INDUCING LIGAND

VV Yurovsky. Department of Medicine, University of Maryland - Baltimore, Baltimore, USA

10.1136/annrheumdis-2001.827

Background Tumour necrosis factor-related apoptosis-inducing ligand (TRAIL), also called Apo-2 ligand, is a member of the TNF family that has been reported to induce apoptosis in a variety of transformed cell lines, as well as in normal human hepatocytes in vitro. Among the family members, TRAIL displays highest homology to CD95 ligand, receptor of which may not only mediate apoptosis of $\mathrm{T}$ cells, but also mediate the proliferation of normal human fibroblasts. Considering structural and functional similarities between TRAIL and CD95 ligand, we examined the effects of soluble TRAIL on normal human lung fibroblasts. Collagen a2(I) mRNA expression in fibroblasts was measured by RT-PCR, with ribosomal protein S9 as an internal standard. Normalised collagen mRNA expression was increased in fibroblasts stimulated with TRAIL for 1 or 7 days, with peak response ( $>5$-fold increase) at $10 \mathrm{ng} / \mathrm{ml}$ TRAIL. The increased expression of collagen a2(I) gene was confirmed by cDNA microarray that also revealed 72 other genes with expression level increased and 108 genes with expression level decreased ? 2.2-fold in comparison with quiescent fibroblasts. There was little, if any, effect of TRAIL on fibroblast proliferation. In addition, the expression of TRAIL was found in $\mathrm{CD} 8^{+} \mathrm{T}$ cell clones that had undergone oligoclonal expansion in the lungs of patients with systemic sclerosis (scleroderma) and were able to stimulate collagen production in lung fibroblasts in vitro. These data suggest that TRAIL can enhance collagen production by fibroblasts that are resistant to TRAIL-induced apoptosis.

Objectives

Methods

Results

Conclusion

\section{THU0031 HIGH SYNOVIAL FLUID AND SERUM IL-18 LEVELS IN PATIENTS WITH RHEUMATOID AND PSORIATIC ARTHRITIS}

V Pittoni, M Bombardieri, A Spadaro, A Ossandon, FR Spinelli, E Taccari, V Riccieri, T Rinaldi, C Alessandri, G Valesini. Terapia Medica, Unità Di Reumatologia, Università "La Sapienza", Rome, Italy

\subsection{6/annrheumdis-2001.828}

Background IL-18 is a recently identified cytokine with a broad array of proinflammatory actions. Il-18 is expressed at sites of chronic inflammation in human autoimmune diseases and is thought to play a pathogenetic role especially in those immunemediated diseases with a predominant Th1 cytokine profile.

Objectives The aim of this study was to measure IL-18 levels in sera and synovial fluids (SF) of patients with rheumatoid arthritis (RA), psoriatic arthritis (PsA) and osteoarthritis (OA).

Methods Eighty-one patients were studied, of which thirty-three with RA (11M, 22F; mean age $54.7 \pm 12.8$ yrs), twenty-nine 